\title{
Multiwavelength studies of the gas and dust disc of IRAS $04158+2805^{\star}$
}

\author{
A. M. Glauser ${ }^{1}$, F. Ménard ${ }^{2}$, C. Pinte ${ }^{4}$, G. Duchêne ${ }^{23}$, M. Güdel ${ }^{1}$, J.-L. Monin ${ }^{2}$, and D. L. Padgett ${ }^{5}$ \\ 1 Paul Scherrer Institut, 5232 Villigen PSI, Switzerland \\ e-mail: glauser@phys.ethz.ch; adrian.glauser@psi.ch \\ 2 Laboratoire d'Astrophysique de Grenoble, CNRS/UJF UMR 5571, BP 53, 38041 Grenoble Cedex 9, France \\ Astronomy Department, UC Berkeley, Berkeley CA 947209-3411, USA \\ School of Physics, University of Exeter, Stocker Road, Exeter EX4 4QL, UK \\ Caltech-JPL/IPAC, Mail Code 100-22, Pasadena, CA 91125, USA
}

Received 24 May 2006 / Accepted 14 April 2008

\section{ABSTRACT}

\begin{abstract}
We present a study of the circumstellar environment of IRAS $04158+2805$ based on multi-wavelength observations and models. Images in the optical and near-infrared, a polarisation map in the optical, and mid-infrared spectra were obtained with VLT-FORS1, CFHT-IR, and SPITZER-IRS. Additionally we used an X-ray spectrum observed with Chandra. We interpret the observations in terms of a central star surrounded by an axisymmetric circumstellar disc, but without an envelope, to test the validity of this simple geometry. We estimate the structural properties of the disc and its gas and dust content. We modelled the dust disc with a 3D continuum radiative transfer code, MCFOST, based on a Monte-Carlo method that provides synthetic scattered light images and polarisation maps, as well as spectral energy distributions. We find that the disc images and spectral energy distribution narrowly constrain many of the disc model parameters, such as a total dust mass of $1.0-1.75 \times 10^{-4} M_{\odot}$ and an inclination of $62^{\circ}-63^{\circ}$. The maximum grain size required to fit all available data is of the order of 1.6-2.8 $\mu \mathrm{m}$ although the upper end of this range is loosely constrained. The observed optical polarisation map is reproduced well by the same disc model, suggesting that the geometry we find is adequate and the optical properties are representative of the visible dust content. We compare the inferred dust column density to the gas column density derived from the X-ray spectrum and find a gas-to-dust ratio along the line of sight that is consistent with the ISM value. To our knowledge, this measurement is the first to directly compare dust and gas column densities in a protoplanetary disc.
\end{abstract}

Key words. stars: circumstellar matter - stars: pre-main-sequence - stars: individual: IRAS $04158+2805$ - stars: formation stars: planetary systems: protoplanetary discs

\section{Introduction}

Accretion discs are key elements in star and planet formation scenarios. They provide the material for accretion leading to star and planet building, they provide the energy and material for launching jets, and they are the medium through which angular momentum is transported away. Knowing their geometrical and physical properties is important for understanding these processes and their evolution.

Large-scale surveys have been performed to search for discs around young low-mass pre-main sequence stars (e.g., Stapelfeldt et al. 2003; Schneider et al. 2005), the so-called $\mathrm{T}$ Tauri stars. A few tens of discs have been imaged and, for many of them, images are available over a progressively broader wavelength range, enabling deeper studies of the disc properties.

However, the properties of discs around more massive stars and, of concern here, around the lower mass late $\mathrm{M}$ dwarfs and brown dwarfs remain poorly known, because images for these discs are still extremely rare. As a consequence, our knowledge

\footnotetext{
* Based on observations obtained at the Canada-France-Hawaii Telescope (CFHT) which is operated by the National Research Council of Canada, the Institut National des Sciences de l'Univers of the Centre National de la Recherche Scientifique of France, and the University of Hawaii. Based also on data collected at ESO/VLT during observation program 68-C.0171.
}

of the circumstellar environment of these objects is based solely on spectral energy distribution (SED) fitting.

In this paper we present a study of IRAS $04158+2805$, a lowmass star located near the substellar boundary. The classification of IRAS $04158+2805$ varies in the literature, with most authors agreeing on a late spectral type, implying a very low stellar mass. The recent paper by Luhman (2006) concludes that its spectral type is consistent with a low mass star close to the brown dwarf limit. IRAS $04158+2805$ is located at a distance of $140 \mathrm{pc}$, in the L1495 east dark cloud, which is part of the Taurus molecular cloud complex. It is surrounded by an extended reflection nebulosity seen in scattered light. It propels a well-collimated, ionised atomic jet seen in $\mathrm{H} \alpha$ that extends at least out to 60 arcsec to the north. The object is probably located in the foreground of the large reflection nebulosity illuminated by V892 Tau, one of the rare Herbig Ae stars of the Taurus cloud, because the disc of IRAS $04158+2805$ appears in silhouette over the reflection nebulosity. This is the only such case in Taurus to our knowledge.

In this paper, we apply an axisymmetric, inclined-disc model to our data, fitting near-infrared images, near- and mid-infrared photometry, mid-infrared spectroscopy, millimeter photometry, and near-infrared polarimetry to derive disc geometry, disc mass, and dust composition. The large size of the disc derived from the near-infrared images (radius of approximately 8 arcsec in the $I$ band, corresponding to $1120 \mathrm{AU}$ at a distance of $140 \mathrm{pc}$ ) is 

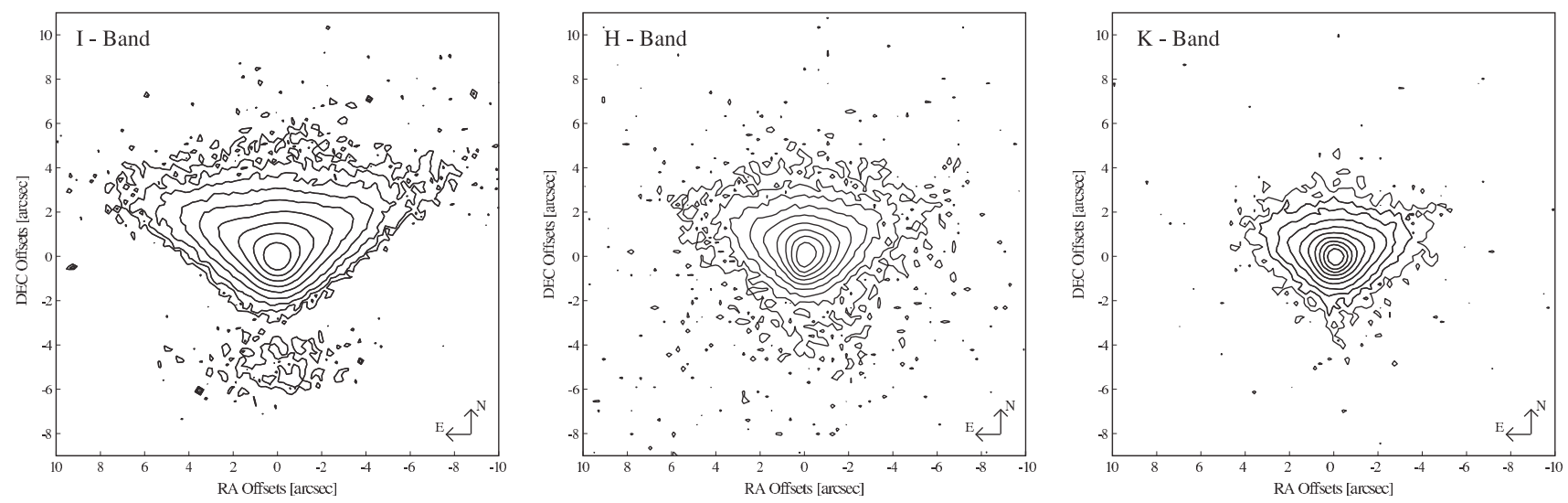

Fig. 1. $I$-, $H$ - and $K$-band contour plots of IRAS $04158+2805$. The contour lines are on the levels $I_{\max } \cdot 2^{-n}$ with $n=1, \ldots, 9$ or 10 for the $K$-band image respectively.

intriguing, especially around a star of such a low mass. We will show that the data are compatible with an inclined disc without any need to invoke a larger envelope. The paper is organised as follows. Section 2 presents the observations which are further discussed in Sect. 3. In Sect. 4, we discuss the modelling of the dust disc. We estimate the structural model parameters of the circumstellar disc and we discuss the quality and the uniqueness of the solution found in Sect. 5. We present our conclusions in Sect. 6.

\section{Observations and data reduction}

\subsection{Optical imaging and polarimetry}

\subsubsection{Observations}

IRAS $04158+2805$ was observed on December 12, 2001 in the I-band with the FORS1/IPOL instrument. The weather conditions were good and the seeing was measured between 0.9 and $1^{\prime \prime}$ over the observation period. The total field of view (FOV) of FORS1/IPOL is 6.8 $\times 6.8$ in the Standard Resolution mode with a focal scale of 0.2 /pixel. Polarimetry was performed by inserting a Wollaston prism in the beam. The prism splits the incident light beam into two separate beams of orthogonal polarisation states, the so-called ordinary $(o)$ and extraordinary $(e)$ beams. A stepped half-wave plate retarder was placed at the entrance of the incident beam and was rotated by steps of 22.5 degrees. The separation of the two $o$ - and $e$-beams on the CCD is performed by the Wollaston prism and overlap of the two beams is avoided by inserting a 9 -slit focal mask. Each slit in the mask provided a $\sim 20^{\prime \prime} \times 6$ 6.8 FOV. Images of IRAS $04158+2805$ were recorded at 8 retarder positions with an integration time of 3 minutes per frame. The images were then combined to yield the Stokes parameters $I, Q$ and $U$.

\subsubsection{Data reduction pipeline}

A dedicated data reduction pipeline was written using NOAO/IRAF (see, e.g., Monin et al. 2006). The images are first corrected for bias and bad pixels, and then flat-fielded. In the next step, the images went through a polarisation extraction routine in which the normalised flux difference between the ordinary and extraordinary images is calculated for every pixel of the image, and a Fourier series was computed to derive the Stokes parameter $I, Q$ and $U^{1}$ : We first computed the normalised flux difference $F$ from the target flux $f$ :

$$
F\left(\theta_{i}\right)=\frac{f^{o}\left(\theta_{i}\right)-f^{e}\left(\theta_{i}\right)}{f^{o}\left(\theta_{i}\right)+f^{e}\left(\theta_{i}\right)} .
$$

Thereafter we computed $Q$ and $U$ :

$Q=\sum_{i=0}^{N-1} \frac{2}{N} F\left(\theta_{i}\right) \cos \left(4 \theta_{i}\right) \quad U=\sum_{i=0}^{N-1} \frac{2}{N} F\left(\theta_{i}\right) \sin \left(4 \theta_{i}\right)$

The absolute errors were estimated by using two independent methods: first, from the photon noise on the $e$ - and $o$-beams separately, and then propagating the errors in the calculations of $Q$, $U, P$ and $\Theta$; second, by measuring the standard deviation on the 8 images from the half-wave plate rotation. Both methods gave consistent results, of order $0.3 \%$. The final intensity map is presented in Fig. 1.

\subsubsection{Instrumental polarisation}

We estimated carefully the instrumental polarisation at the centre of the FORS1 field by measuring nearby (i.e., high proper motion) unpolarised targets. We observed GJ 781.1 and GJ 2147, two high proper motion stars. Because the immediate solar neighbourhood is remarkably devoid of dust (e.g., Leroy 1993, 1999) the interstellar polarisation of nearby stars can be considered null. The average of 4 measurements on both GJ objects gives $P_{\text {inst }}=0.02 \% \pm 0.03 \%$. We therefore believe that FORS1/IPOL instrumental polarisation is very low on-axis, well below $0.1 \%$ at the centre of the field, and we did not attempt to remove it from the measurements.

On the other hand, Patat \& Romaniello (2006) showed that FORS1 presents a spatially variable instrumental polarisation component. This component follows a radial pattern with an intensity scaling as $0.06 r^{2}$ (in $\%$ if $r$ is in arcmin), ranging from $0.1 \%$ in the central region, one arcmin in radius, to $1 \%$ at the edge of the FOV. First, the absolute value of the instrumental polarisation is low $(0.23 \%$ at the position of the source) compared to the observed polarisation of IRAS $04158+2805$ which is always higher than $3 \%$. Second, since the source is small compared to the FOV, we decided to neglect the spatial variation of the instrumental polarisation on the FORS1 detector because it does not vary significantly across the object.

\footnotetext{
1 See the FORS user manual at http://www.eso.org. The polarisation level, $P$, is obtained by calculating $P=\sqrt{Q^{2}+U^{2}} / I$ and the position angle, $\Theta$, by calculating $\Theta=1 / 2 \arctan (U / Q)$.
} 


\subsection{Near-infrared imaging}

On October 29 and 30, 2001, we used the near-infrared CFHT-IR camera (Starr et al. 2000) at the Canada-FranceHawaii Telescope to obtain $H$ - and $K$-band images of IRAS $04158+2805$ with a pixel scale of $0.211 /$ pixel and a total FOV of 3.6. Conditions were non-photometric and the seeing during the observations was 0.65 at $K$-band and $0 . ' 9$ at $H$-band, as measured from the average FHWM of several unresolved point sources located in the FOV. With each filter, two series of 10 jittered images were obtained in separate sets. Each set of images was first reduced as an independent dataset in the following manner. All images were median-combined to create a sky frame, which was subtracted from each image. The images were then flat-fielded, registered based on the location of a bright point source in the field and median-combined. The two independent images per filter resulting from this procedure were then averaged together to produce the final images presented in Fig. 1.

\subsection{Mid-infrared spectroscopy}

We use archival data of the SPITZER Infrared Spectrograph (IRS, see, e.g., Houck et al. 2004) observation from March 4, 2004 (program request 3534848 ) which was done in the spectral mapping mode with the two low resolution channels: Short-Low (SL; 5.2-14 $\mu \mathrm{m}, \lambda / \Delta \lambda \sim 90$ ) and Long-Low (LL; 14-38 $\mu \mathrm{m}$, $\lambda / \Delta \lambda \sim 90$ ). The mapping mode ( 3 exposures across the target for each slit and 2 nodding positions each) was chosen due to the small mispointing of SPITZER in the early mission. Therefore, the flux of the object is separated in two or three observations. To recover the photometric information, all three parallel exposures were summed for the SL while the overlap of the FOV of the LL-slit is such that the full flux is extractable by summing the first and last exposures.

We used the final products from the Spitzer Science Center's IRS data-reduction pipeline (post-BCD). To allow a background subtraction the two nodding observations were used to subtract them from each other. The data extraction was done with the Spitzer IRS Customer Extractor software (SPICE) provided by the Spitzer Science Center.

\subsection{X-ray spectroscopy}

IRAS $04158+2805$ was serendipitously observed by the Chandra X-ray Observatory in a field pointing at the L1495 East dark cloud around V410 Tauri. The observation was performed with ACIS-S on March 7, 2002 between 6:16 UT and 11:45 UT, with a useful total exposure time of approximately $17700 \mathrm{~s}$. IRAS $04158+2805$ was located 11'.6 off-axis on the ACIS-S chip $\mathrm{S} 1$. This resulted in a rather distorted, extended point spread function (PSF) with considerable background contributions. The data were, however, sufficiently clean to extract a useful spectrum (see Fig. 2).

We reduced all ACIS-S data using standard analysis techniques following the Chandra CIAO Science Threads ${ }^{2}$. Specifically, we flagged bad pixels, applied CCD charge transfer inefficiency corrections to create a so-called level2 events file. We extracted source counts from a circular area with a radius of 27.' 6 around the source. To define a background spectrum, a source-free circular area with a radius of $98 . \prime 4$ was extracted from the same chip. The counts were binned into a source and a

\footnotetext{
${ }^{2}$ http://cxc.harvard.edu/ciao/threads/
}

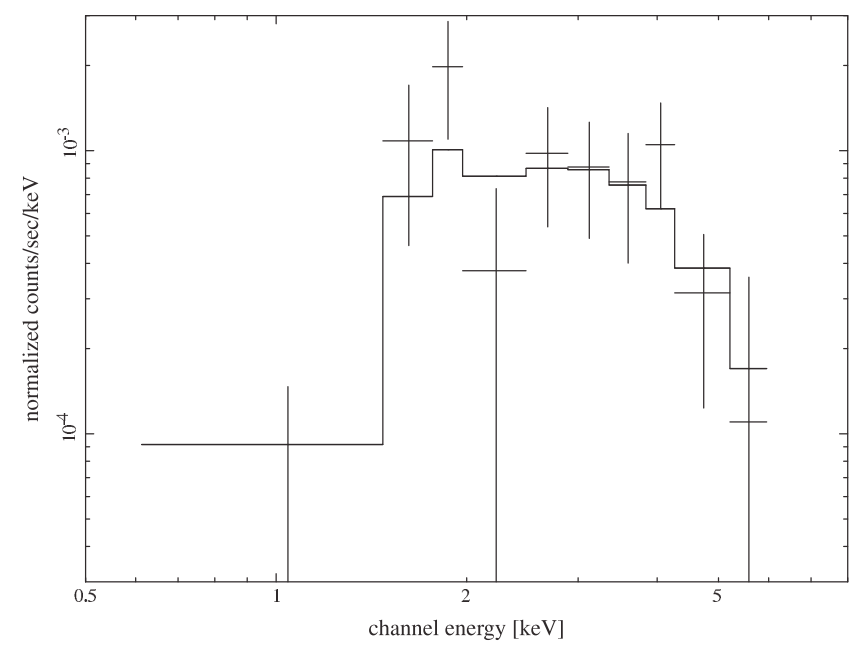

Fig. 2. X-ray spectrum of IRAS04158+2805 observed with Chandra.

background spectrum. Appropriate responses were created using the $m k r m f$ task, and the ancillary file was obtained from mkarf.

\section{Results}

\subsection{Imaging}

Contour plots of IRAS $04158+2805$ at $I$-, $H$ - and $K$-bands are presented in Fig. 1 . In the $I$-band, the object shows a bipolar reflection nebula geometry. The dark lane, tracing the equatorial plane, is seen in absorption over the background light. It separates a prominent triangular nebulosity located to the North from a low surface brightness elongated counter nebula to the south. The counter nebula is undetected at $H$ - and $K$-bands. At these wavelengths, the triangular shape of the main nebula is still visible, but its extension decreases with increasing wavelength.

The maximum width of the northern nebula is measured to be $15^{\prime \prime} .8,14{ }^{\prime \prime} 1$ and $10{ }^{\prime} 8$ at $I-, H$ - and $K$-band, respectively. The northern nebula has a triangular shape whose opening angle is $\sim 130^{\circ}$ at all wavelengths. Finally, the distance between the peak of the two nebulae at $I$-band is $4.8 \pm 0.2$ arcsec. In Sect. 4.2, we define additional morphological and photometric observables to compare models and observations.

\subsection{Aperture photometry}

To construct the spectral energy distribution of IRAS $04158+2805$ shown in Fig. 3, we use mid-infrared IRAS flux measurements from Kenyon et al. (1990), optical and near-infrared photometry from Kenyon et al. (1990), Strom \& Strom (1994) and from the 2MASS point source catalog. The $1.3 \mathrm{~mm}$ continuum flux is from Motte \& André (2001). Because IRAS $04158+2805$ is extended, we suspect that the 2MASS photometry may be underestimated since these fluxes are listed in the 2MASS All-Sky Point Source Catalogue (PSC) and were obtained by PSF fitting. To check this point, we extracted the photometry from our own near-infrared images by using three point-like sources that have 2 MASS photometry and use them as relative photometric standards. We obtained $K=11.04$ and $H=12.10$ for IRAS $04158+2805$, with estimated uncertainties of $0.05 \mathrm{mag}$. For the $H$-band this is $0.28 \mathrm{mag}$ brighter than the 2MASS photometry and $0.13 \mathrm{mag}$ for the $K$-band. While the brightness profile of IRAS $04158+2805$ is peaked in the near infrared and may account for this discrepancy, we note 


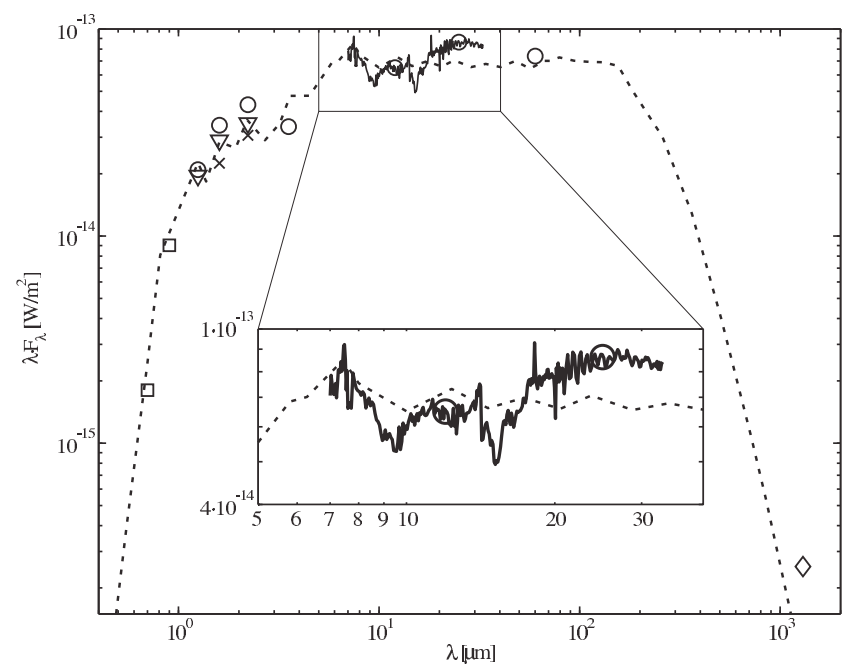

Fig. 3. Modeled SED (dashed line) compared to observed fluxes: IRS spectrum (solid line); Strom \& Strom (1994, squares); Kenyon et al. (1990) and Kenyon \& Hartmann (1995, circles); Luhman \& Rieke (1998) and Luhman (2000, crosses); Motte \& André (2001, diamonds); this paper, see Sect. 3.2 (triangle). The error bars of the photometric fluxes are smaller than their symbols and therefore not drawn.

that from our images we find a difference of only 0.03 mag in the photometry when two apertures of $4 .{ }^{\prime \prime} 2$ and $10 . ' 6$ are used. Therefore, we attribute the difference in photometry of IRAS $04158+2804$ between 2 MASS and our images to intrinsic variability of the source rather than an aperture size too small in 2MASS.

\subsection{Mid-infrared spectroscopy}

The SPITZER-IRS spectrum shows absorption features at $\sim 10 \mu \mathrm{m}$ and $\sim 15.2 \mu \mathrm{m}$. Both can be identified as silicates and carbon dioxyde ice, respectively (see Fig. 3). Similar features are seen for example in low-mass protostars, where the cold envelope and/or outer disc cause the absorption (e.g., Watson et al. 2004). In IRAS $04158+2805$, it is interesting to note that the silicate absorption is maximum slightly short of 10 microns, around 9.5 microns. This is an indication for small dust grains made of either amorphous or crystalline material such as enstatite (Schegerer et al. 2006). We did not attempt to fit the exact shape of the feature and cannot conclude about the exact mineralogy of the grains. It is also interesting to find $\mathrm{CO}_{2}$ ice signatures in absorption in a source where the disc appears to dominate the SED (see Sect. 4.2.3), but the high inclination, nearly edge-on, probably provides the needed column density along the line of sight. However, it is beyond the scope of this paper to discuss quantitatively the IRS spectrum. We will do so in a forthcoming paper.

We extract the fluxes at $12 \mu \mathrm{m}$ and $25 \mu \mathrm{m}$ and compare with IRAS fluxes published in Kenyon et al. (1990). The difference is of the order of $0.5 \%$ at $12 \mu \mathrm{m}$ and $1.3 \%$ at $25 \mu \mathrm{m}$ and is negligible therefore.

\subsection{I-Band polarimetric imaging}

The light from the northern photometric nebulosity centre has a linear polarisation of $3.3 \pm 1.2 \%$, averaged within a region of 2 arcsec (10 pixels) in diameter. Since neighbouring objects do not show a significant polarisation rate, this is an indication that the peak in the nebula is not arising from stellar photons seen directly but, instead, from scattered light. The polarisation is maximum at the corners of the triangular northern nebula and has values between $35 \%$ and $41 \%$ (see Fig. 4). For this determination we use the highest "reliable" value. We decided to use only those pixels for which the polarisation rate topology shows a certain smooth behaviour. This was quantified by determining the standard deviation of the polarisation rate of each pixel and its 4 neighbours. If the standard deviation is higher than $20 \%$ (typical noise level), the pixel is not used.

\section{5. $X$-rays}

The Chandra ACIS X-ray spectrum of IRAS $04158+2805$ is shown in Fig. 2. The spectrum was binned such that the minimum number of counts per bin was 10 before background subtraction. The total number of counts in the $0.5-6 \mathrm{keV}$ range was 100 , which allowed for only a rather simplistic spectral model that is, however, sufficient to derive useful estimates of the absorption column density to the X-ray source. The source spectrum is obviously very strongly absorbed, with essentially no counts detected below $1.5 \mathrm{keV}$. The X-ray source is hard enough to produce counts up to $6 \mathrm{keV}$ in the observation.

We fitted the spectral data in XSPEC (Arnaud et al. 1996), using the vapec collisional-ionization equilibrium model and a photoelectric absorption component, the latter essentially being defined by the hydrogen column density $N_{\mathrm{H}}$ between observer and source. We first fitted the spectrum with a single thermal component, assuming that all element abundances are at 0.3 times the solar values, referring to the solar abundances of Anders \& Grevesse (1989). This corresponds to the elemental composition typically measured in coronae of magnetically active stars (Güdel 2004).

We found a best-fit temperature of $5.8 \mathrm{keV}$ or $6.7 \times 10^{7} \mathrm{~K}$, and a best-fit value for $N_{\mathrm{H}}$ of $3.3_{-1.4}^{+2.2} \times 10^{22} \mathrm{~cm}^{-2}$, where the error range refers to the $68 \%$ confidence limit. Given that the spectrum reveals only the hard end of the entire soft X-ray spectrum, the fit may be biased by fitting a hot component only. This bias may be relieved somewhat by fitting a continuous distribution of emission measures $\left(\mathrm{EMD}^{3}\right)$. We adopted an EMD with a prescribed shape such as found for less-absorbed T Tauri stars, described in more detail by Telleschi et al. (2007). The EMD model essentially consists of two power laws on each side of a peak. Neither the location of the EMD peak nor the power-law slope toward higher temperatures were well constrained, but the results for $N_{\mathrm{H}}$ were robust and converged to $N_{\mathrm{H}}=3.6_{-1.0}^{+2.5} \times 10^{22} \mathrm{~cm}^{-2}$.

To summarise these two approaches, irrespective of the uncertainties in the intrinsic X-ray spectrum of IRAS 04158+2805, we find that the X-rays, which are presumably emitted in the corona surrounding the central star, are attenuated by a gas column density of $N_{\mathrm{H}} \approx 3.5 \times 10^{22} \mathrm{~cm}^{-2}$, with a one sigma range of $(1.9-6.1) \times 10^{22} \mathrm{~cm}^{-2}$.

\section{Modelling of the dust disc}

In this section we explore the parameter space of a simple model comprising the stellar photosphere and a power-law disc (see Sect. 4.1.1 below) to explain the observations of IRAS $04158+2805$ and its associated reflection nebulosity.

\footnotetext{
3 EMD defines the the emission measure $E M=n_{\mathrm{e}} n_{\mathrm{H}} V$ as a function of the temperature, where $n_{\mathrm{e}}, n_{\mathrm{H}}$ are electron and hydrogen densities, respectively, and $V$ is the volume.
} 

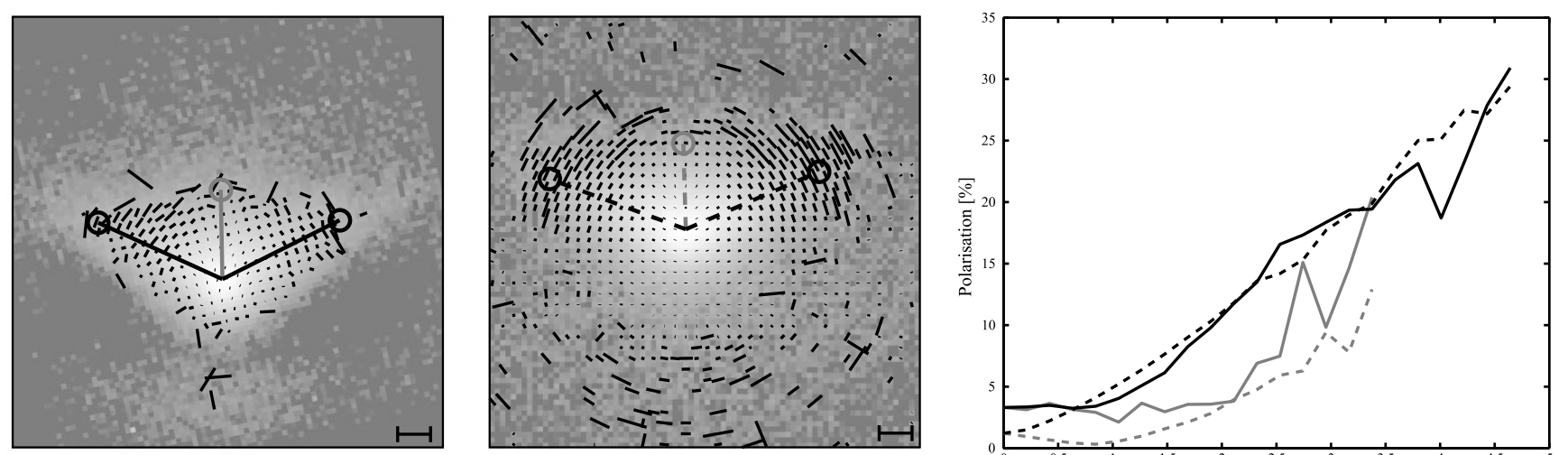

Fig. 4. Polarisation maps of the observed (left panel) and modelled (middle panel) nebula. The vector length for $100 \%$ polarisation is indicated by the bar in the lower right corner. Right panel: comparison of the polarisation level as a function of position in the observed (solid) and modelled (dashed) nebula. These curves are estimated along the ridges (black curves) and symmetry axis (gray) of the nebula.

\subsection{Modelling technique}

Synthetic images, polarisation maps and spectral energy distributions are computed using MCFOST, a 3D radiative transfer code based on the Monte-Carlo method. MCFOST is described in details in Pinte et al. (2006). MCFOST solves the full polarised radiative transfer in dusty environment. It includes multiple scattering, passive heating of the dust disc and thermal reemission by the dust to produce synthetic images in all four Stokes parameters at any wavelength, as well as SEDs. The dust temperature, the same for all grain sizes, is calculated assuming local thermal equilibrium.

\subsubsection{System geometry}

We consider a flared density structure with a Gaussian vertical profile $\rho(r, z)=\rho_{0}(r) \exp \left(-z^{2} / 2 h(r)^{2}\right)$, valid for a well-mixed vertically isothermal, hydrostatic, non-self-gravitating disc. We use power-law distributions for the surface density $\Sigma(r)=$ $\Sigma_{0}\left(r / r_{0}\right)^{\alpha}$ and for the scale height $h(r)=h_{0}\left(r / r_{0}\right)^{\beta}$ where $r$ is the radial coordinate in the equatorial plane and $h_{0}$ is the scale height at the radius $r_{0}=50 \mathrm{AU}$. The disc extends from an inner cylindrical radius $R_{\text {in }}$ to an outer limit $R_{\text {out }}$. The central star is represented by a point-like, isotropic source of photons.

\subsubsection{Dust properties}

We consider homogeneous spherical grains and we use the dielectric constants described by Mathis \& Whiffen (1989) in their model A, which accounts for the interstellar extinction law. Grain sizes are distributed according to a power-law $n(a) \propto a^{-3.7}$ with $a_{\min }$ and $a_{\max }$ being the minimum and maximum grain radii. The interstellar values from Mathis \& Whiffen (1989) are $a_{\min }=0.005 \mu \mathrm{m}$ and $a_{\max }=0.9 \mu \mathrm{m}$. The mean grain density is $0.5 \mathrm{~g} \mathrm{~cm}^{-3}$ as a consequence of the high porosity (80\%) of the grains. In this work, $a_{\max }$ is considered as a free model parameter to fit for. Extinction and scattering opacities, scattering phase functions and Mueller matrices are calculated using Mie theory. The dust and gas are assumed to be perfectly mixed and the grain properties are taken to be independent of position within the disc.

\subsection{Data fitting}

In the process of extracting the disc geometric parameters from the observations, we first attempt to fit the $I$-, $H$ - and $K$-band images simultaneously with a single disc model. This is presented in 4.2.1. We use $a_{\max }, m_{\text {dust disc }}, \beta, \alpha$ and the inclination as free model parameters. The $I$ band image suggests a maximum disc radius of 8 arcsec, which corresponds to 1120 AU at a distance of $140 \mathrm{pc}$. There must also be an inner disc radius, which is typically at a few tenths of an AU. These radii are poorly constrained by our $I$-, $H$, and $K$-band images; we therefore fix them at the given values (inner radius is set to $0.5 \mathrm{AU}$ ), which will have little effect on our results. Since we do not implement background illumination into our model, the appearance of the dark lane and the counter nebula is expected to be slightly different in the model.

We computed about 12000 models covering a wide range of the free parameters. A handful of viable solutions remain after the image-fitting process (in Sect. 4.2.2). We explore the relative merits of these few solutions by calculating their respective SEDs (in Sect. 4.2.3), and I-band polarisation map (in Sect. 4.2.5) for comparison with the data.

\subsubsection{Image processing and extraction of observables}

To permit a direct comparison of the observed and modelled images, observational effects must be added to the model images. First of all, the pixel size of the model output is therefore chosen to match the scale of the observation ( 0 '200/pixel in the optical, 0.'211/pixel in NIR). The model images must be convolved with the observed PSF and scaled to the same peak value as the observed images which allows an addition of Poisson and background noise to yield the same signal-to-noise ratio.

Having two comparable images, it is now possible to quantitatively compare the model and observed images. To avoid potential biases induced by asymmetries in the disc structure and the ignorance of the background illumination of the object and to circumvent the intrinsically noisy nature of Monte Carlo images, we decided to use a "quality metric" based on geometrical observables of the nebula rather than an image-wide pixelto-pixel goodness-of-fit estimate. These observables, which are detailed below and illustrated in Fig. 5 are chosen to describe the width of the nebula, its triangular shape and the counter nebula behaviour relative to the northern nebula. They are extracted with the same routines in the observed and modelled images, after further smoothing the images with a Gaussian (width $\sigma=1$ pixel). We first define the outer "ridges" of the triangular nebula. To allow this, we calculate contour lines at the flux levels $I_{n}=I_{\max } \cdot 2^{-n}$ with $n=1,2, \ldots, N$, while $N$ defines the last contour line at flux level higher than the noise. The 


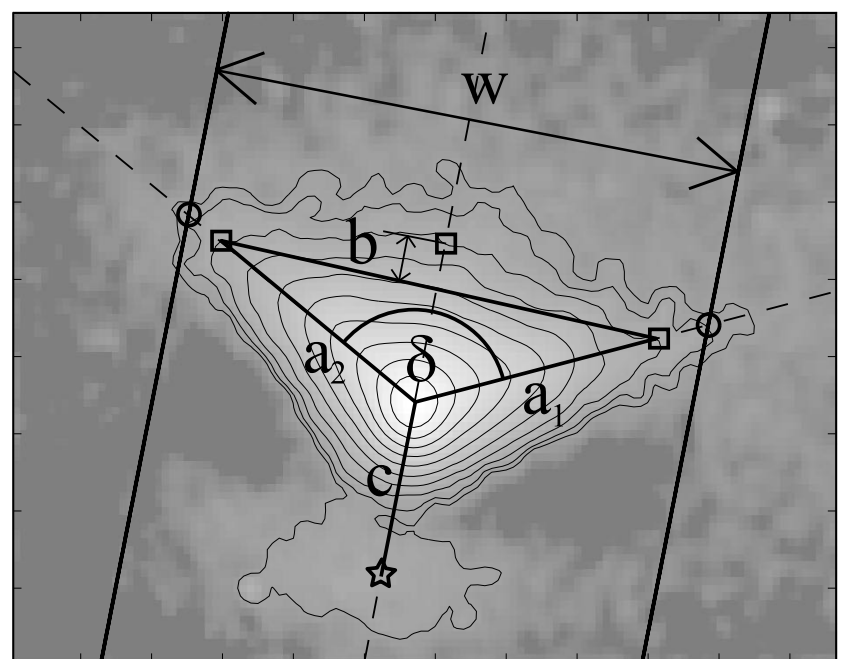

Fig. 5. Definition of geometrical observables $w, a, b, c$ and $\delta$. The contour lines are shown for the levels $I_{\max } \cdot 2^{-n} n=1 \ldots 10$. The two ridges and the symmetry axis are drawn as dashed lines. Their intersection points with the contour lines of the flux level $I=I_{\max } \cdot 2^{-8}$ are shown as boxes and $I=1 \cdot \sigma_{\text {noise }}$ as circles, respectively. The counter nebula peak is shown as a star.

ridges (labelled with $a_{1}$ and $a_{2}$ in Fig. 5) are then defined by two lines that cross those points on each contour that are most distant from the central peak. We derive these lines as follows: We represent the contours in polar coordinates as $r_{n}(\phi)$ where $r$ is the distance from the central peak and $\phi$ is the angle from North. These polar functions are then normalised and averaged $\left(r(\phi)=n^{-1} \sum_{n} r_{n}(\phi) / \int_{0}^{2 \pi} r_{n}\left(\phi^{\prime}\right) \mathrm{d} \phi^{\prime}\right)$. The direction of the two lines are described by the two values of $\phi$ where $r(\phi)$ has a maximum. Thereafter, the opening angle $\delta$ between the two ridges was measured. The symmetry axis of the nebula corresponds to their bisecting line.

The width of the nebula is defined as the distance $w$ between the two intersection points of the ridges with the contour at the level of a signal-to-noise ratio equal to 1 (shown in Fig. 5 as circles).

The next observable describes quantitatively whether the nebula looks triangular or round. This observable, which we call the triangularity, $t$, is determined for each contour level. In Fig. 5 an example is shown for the contour level $n=8$. The two ridges and the symmetry axis are intersected with the contour line (shown as squares in the figure). The distance between the intersection point of the symmetry axis with the contour line and the triangle spanned by the peak and the two intersection points of the contour line with the ridges is called $b$. The triangularity is then defined as

$$
t=\frac{b}{a(1-\cos (\delta / 2))}
$$

where $a$ is the average of $a_{1}$ and $a_{2}$. This parametrisation was chosen to describe a perfectly triangular nebula for $t=0$ and a perfect circle for $t=1$. For the model-to-data comparison, we fitted the most triangular contour line, which describes best the shape of the outer nebula.

Finally, the counter nebula is described by the peak-to-peak distance $c$, and the contrast is defined as the ratio between the maximum brightness of the nebula and the counter nebula.
Table 1. Weights $g_{i, \lambda}$ of the pseudo- $\chi^{2}$ function (4).

\begin{tabular}{lccccc}
\hline \hline & $\delta$ & $w$ & $t$ & $c$ & Contrast \\
\hline$I$ & $1 / 9$ & $3 / 9$ & $3 / 9$ & $1 / 9$ & $1 / 9$ \\
$H$ & $1 / 7$ & $3 / 7$ & $3 / 7$ & 0 & 0 \\
$K$ & $1 / 7$ & $3 / 7$ & $3 / 7$ & 0 & 0 \\
\hline
\end{tabular}

Table 2. Disc model parameters for the best-fitting model and their acceptable range.

\begin{tabular}{lccc}
\hline \hline Parameter & $\begin{array}{c}\text { Explored } \\
\text { range }\end{array}$ & $\begin{array}{c}\text { Best } \\
\text { model }\end{array}$ & $\begin{array}{c}\text { Acceptable } \\
\text { range }\end{array}$ \\
\hline$a_{\max }[\mu \mathrm{m}]$ & $0.5-4.0$ & 2.0 & $1.6-2.8$ \\
$M_{\text {dust disc }}\left[10^{-4} M_{\odot}\right]$ & $0.25-3.0$ & 1.5 & $1.0-1.75$ \\
$R_{\text {out }}[\mathrm{AU}]$ & not varied & 1120 & - \\
$R_{\text {in }}[\mathrm{AU}]$ & not varied & 0.5 & - \\
$\beta$ & $1.0-1.3$ & 1.20 & $1.15-1.21$ \\
$\alpha$ & $-2.0--0.1$ & -1.0 & $-1.5--0.5$ \\
$h_{0}(r=50 \mathrm{AU})[\mathrm{AU}]$ & $5.0-12.0$ & 8.0 & $7.8-8.2$ \\
incl. $\left[\left[^{\circ}\right]\right.$ & $0.0-90.0$ & 62.7 & $62.1-63.25$ \\
\hline
\end{tabular}

\subsubsection{Image fitting}

For a given set of free model parameters $(\alpha, \beta, \ldots)$, we use the following quality metric to estimate whether the model actually reproduces the three images of the object. This metric is a pseudo- $\chi^{2}$, defined as follows:

$f^{2}=\sum_{\lambda, i} \frac{\left(o_{i, \lambda}^{\text {model }}-o_{i, \lambda}^{\mathrm{obs}}\right)^{2}}{w_{i, \lambda}} \cdot g_{i, \lambda}$

where $o_{i, \lambda}^{\text {model }}$ and $o_{i, \lambda}^{\text {obs }}$ are the observables with index $i$ (i.e. $w$, $t, c, \delta$, contrast) for a given photometric band $\lambda(I, H, K)$ of the model image and observation image, respectively. The normalisation with $w_{i, \lambda}$, which is determined by the standard deviation $\operatorname{std}\left(\left(o_{i, \lambda}^{\text {model }}-o_{i, \lambda}^{\text {obs }}\right)^{2}\right)$ over the whole explored model parameter space, allows an equitable comparison of different quantities. Additionally, each observable is weighted with $g_{i, \lambda}$. These weights were fine-tuned by hand to equalise the influence of each individual parameter. This weighting is important to allow for a wavelength-dependent contrast parameter (e.g. the counter nebula is not visible in the $H$ and $K$ band, therefore the parameters $c$ and the contrast are useless for these bands). Additionally the use of some parameters provides stronger constraints towards a good fitting solution than others (e.g. the nebula width and triangularity in comparison to the opening angle). The weights are listed in Table 1.

The exploration of the parameter space was performed iteratively. We first ran a series of models with only 2 variable model parameters. Once the best model in the series was identified, we fixed these model parameters and created a new series by varying two other parameters, and so on. The range of the explored parameter space is listed in the second column of Table 2. The first selection of the parameter range was chosen arbitrarily but reasonably. If the model behaviour suggested possible solutions outside the scanned range, the exploration was extended until the model diverged significantly from the observation. Among all these models, only a few provided good fits simultaneously to the images of IRAS $04158+2805$. In addition, we note that fitting the images at each wavelength independently leads to models of similar parameters and quality. The combination of all images in a single fit allows, however, to narrow down the list of possible good models. 

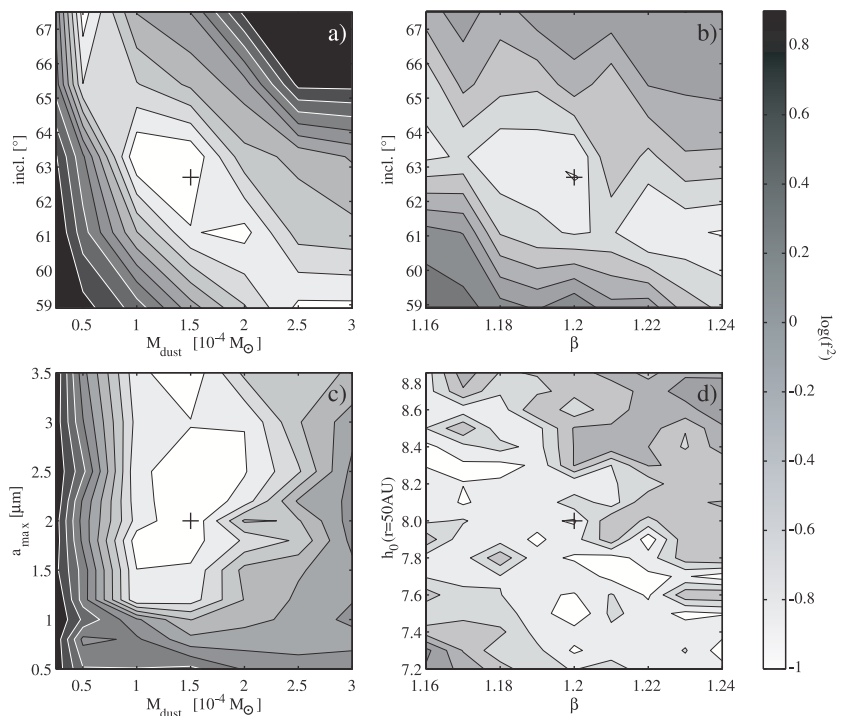

Fig. 6. Examples of projections of $f^{2}$ (Eq. (4)) to different model parameter planes. The contour is plotted in a logarithmic scale. White areas correspond to minimal values of $f^{2}$. The colour range is identical in all four plots. The black cross shows the location of the best model, after the SED fitting.

Figure 6 shows the map of $f^{2}$ as a function of pairs of free model parameters: Dust disc mass vs. inclination (6a), flaring coefficient $\beta$ vs. inclination (6b), dust disc mass vs. maximum grain size (6c), $\beta$ vs. scale height $h_{0}$ (6d). These plots demonstrate the complexity of the search for the minimum since some model parameters are strongly correlated (shown, e.g., in Fig. 6a and b) or show numerous local minima (e.g., Fig. 6d). While Fig. $6 \mathrm{c}$ implies a smooth topology for the $M_{\text {dust }}$ vs. $a_{\max }$ plane, the minimum found at $M_{\text {dust }}=1.5 \times 10^{-4} M_{\odot}$ and $a_{\max }=2 \mu \mathrm{m}$ is only valid for the inclination of $i=63^{\circ}$. At an inclination of $i=61^{\circ}$ we find a minimum with a similar goodness-of-fit with $M_{\text {dust }}=2.5 \times 10^{-4} M_{\odot}$ and $a_{\max }=2.5 \mu \mathrm{m}$. This implies that we cannot find a unique best model by fitting images only. The synthetic images for our best-fitting model, found after the SED fitting process, is presented in Fig. 7 alongside the observed images.

We estimated the valid model parameter range by using one good-fitting parameter set and changed only one parameter until the $f^{2}$ function showed a significant increase or until an eyeball comparison could clearly detect an unsatisfying model. This method does not include an estimation of the general solution range but gives an impression of the validity of the free model parameters at a certain minimum of $f^{2}$.

Additionally we had to set an artificial upper limit for the valid range of $a_{\text {max }}$ : Fig. $6 \mathrm{c}$ shows a valley for $M_{\text {dust }}=1.5 \times$ $10^{-4} M_{\odot}$ and $a_{\max } \geq 2 \mu \mathrm{m}$. This is a consequence of comparing the modelled with the observed images only up to the $K$-band. Therefore, the fit is only mildly sensitive to larger grains, and we set $a_{\max }=2 \mu \mathrm{m}$ with no further consequences for the solution.

Fitting the images for each wavelength individually delivers only very small variations in $a_{\max }$ and disc mass and is consistent with the multi-wavelength solution within the valid model parameter range.

\subsubsection{SED fitting}

To refine the model selection we fit the SED. The modelled SED includes photospheric emission, scattered light and thermal
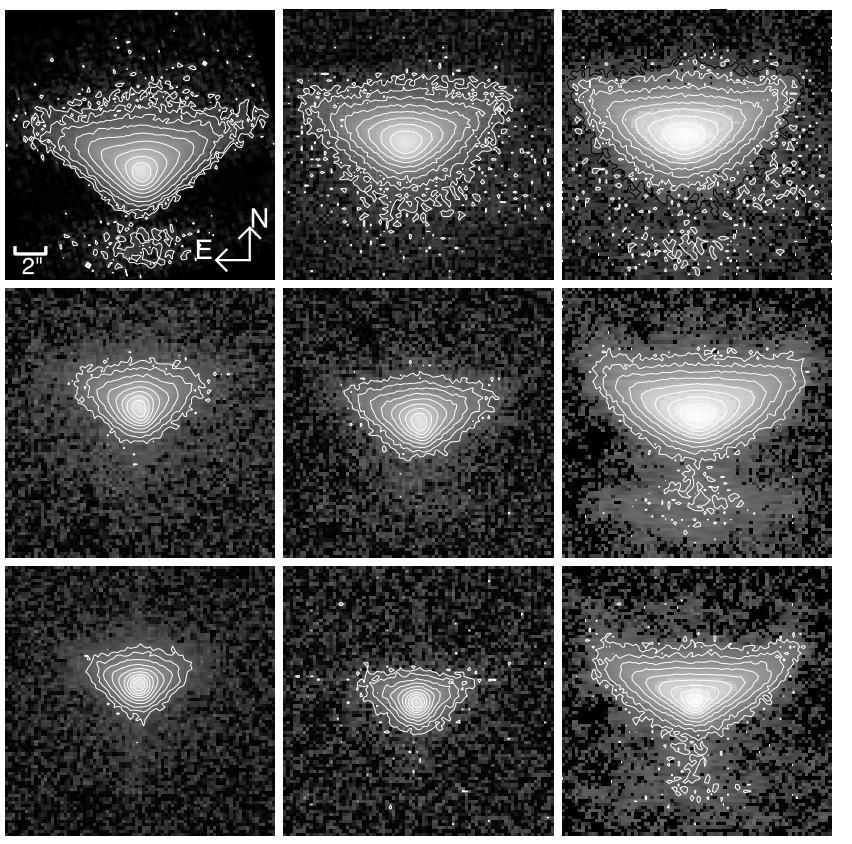

Fig. 7. Comparison between observations (left) and best fitting model (mid, see Table 2) in $I$-band (top), $H$-band (middle) and $K$-band (bottom). For demonstration purposes, the right column shows the images of the best fitting model but with doubled dust disc mass $\left(3 \times 10^{-4} M_{\odot}\right)$.

emission by assuming passive heating of the dust in the disc (Pinte et al. 2006). We use a $3000 \mathrm{~K}$ spectrum from Baraffe et al. (1998) in agreement with a spectral type of a low mass star. For each of the best image-fitting models there are only 2 extra free parameters to the SED fitting: the value of foreground extinction $A_{V}$ and the bolometric luminosity of the central source. The latter is a free parameter since we do not see the source directly. The foreground extinction $A_{V}$ describes the material between the observer and the outer limit of the star-disc system and is added to the model after the radiation transfer calculation.

It turns out that only one model is a simultaneous good fit to the images and to the SED. We consider it our best model in the following (see third column of Table 2). The SED for this model is presented in Fig. 3. It includes $A_{V}=0.5 \mathrm{mag}$ and a stellar luminosity of $\sim 0.4 L_{\odot}$.

Photometric variations are observed for IRAS 04158+2805. It results in a large spread in the SED data at a given wavelength in the optical and NIR (e.g., $1.5 \mathrm{mag}$ in the $I$-band between Strom \& Strom 1994; Luhman 2000). The calculated SED agrees well with the data points and falls within the range of observed variations. The estimated extinction is lower than the value estimated by Strom \& Strom $\left(1994 ; A_{J}=0.7\right)$, Luhman $\&$ Rieke $\left(1998 ; A_{J}=1.5\right)$, and Luhman $\left(2000, A_{H}=1.5\right)$ in the NIR. However these estimations did not take into account the fact that the object's colours are strongly modified by the scattering in the circumstellar material. We therefore believe that our estimate of the foreground extinction is more robust than previous estimates.

\subsubsection{Acceptable parameter range}

The parameters of the best-fitting model are described in Table 2 under "best model". To define the "acceptable range" (see fourth column of Table 2) we calculated series of images from the best model in which only one parameter at a time was varied from its best fit value. This allowed us to define a possible range of 

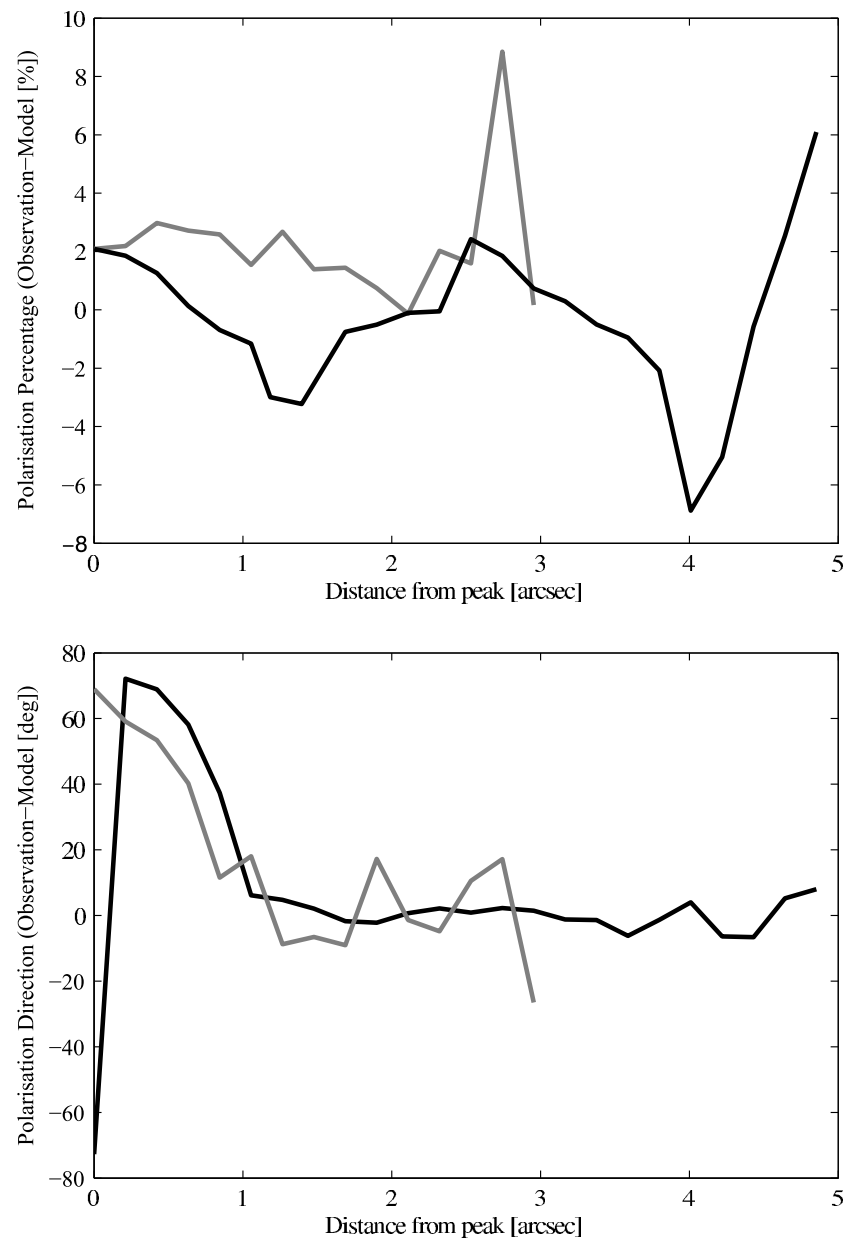

Fig. 8. Difference of observed and modelled polarisation percentage (top) and polarisation direction (bottom) along the ridges (black) and the symmetry line (grey).

solutions, i.e., solutions with values of $f^{2}$ only moderately higher than the best model $\left(f^{2} / f_{\min }^{2} \lesssim 125 \%\right)$ or images that showed no obvious mismatches with the observations by an eyeball comparison. These ranges give a feeling of how tightly each parameter is constrained.

\subsubsection{Using polarisation to confirm the model}

For the best-fitting model we compared the polarisation map with the data. Observational effects are applied to the model polarisation output in a similar manner as for the direct images.

To compare the model with the data, the polarisation values are extracted along the ridges and the symmetry axis of the Northern nebula (see Fig. 4 left and middle panel) and compared as a function of distance from the central source (right panel). The difference of the observed and the modelled polarisation rate and orientation can be found in Fig. 8. The observed polarisation rates are reproduced reasonably well along the ridges by the model $(\Delta P / P \approx 0.3)$ except on the central peak where the model predicts only a $1.2 \%$ polarisation instead of the observed $3.3 \%$. The large difference in polarisation orientation close to the central peak is an artifact from the exact peak placement and has no physical meaning. The upper limit of the observed polarisation is also well reproduced $(\sim 30 \%)$. Along the symmetry axis of the nebula the general trend is also correct, rising from central source to edge, but the model polarisation
Table 3. Extracted observables of the observation and the best-fit model.

\begin{tabular}{lccc}
\hline \hline Observable & Band & Observation & Model \\
\hline \multirow{2}{*}[\operatorname{arcsec}]{} & $I$ & 15.8 & 14.9 \\
& $H$ & 14.1 & 14.0 \\
& $K$ & 10.8 & 12.1 \\
\hline \multirow{2}{*}[^{\circ}]{} & $I$ & 129 & 136 \\
& $H$ & 129 & 133 \\
$t$ & $K$ & 136 & 139 \\
\hline & $I$ & 0.31 & 0.33 \\
& $H$ & 0.44 & 0.33 \\
Contrast & $K$ & 0.53 & 0.23 \\
\hline$c[$ arcsec] & $I$ & 340 & 300 \\
& $H$ & $>1100$ & - \\
& $K$ & $>8700$ & - \\
\hline & $I$ & 4.76 & 4.22 \\
\hline $\left.10^{-14} \mathrm{~W} \mathrm{~m}^{-2} \mathrm{~Hz}^{-1}\right]$ & $0.7 \mu \mathrm{m}$ & $0.18^{1}$ & 0.25 \\
& $0.9 \mu \mathrm{m}$ & $0.90^{1}$ & 1.11 \\
& $1.25 \mu \mathrm{m}$ & $1.96^{2}-2.09^{3}$ & 2.26 \\
& $1.6 \mu \mathrm{m}$ & $2.24^{4}, 2.9^{6}, 3.42^{3}$ & 2.85 \\
& $2.22 \mu \mathrm{m}$ & $3.06^{4}, 3.46^{6}, 4.31^{3}$ & 3.61 \\
& $12 \mu \mathrm{m}$ & $1.19^{2}$ & 4.75 \\
& $25 \mu \mathrm{m}$ & $6.50^{3}$ & 7.19 \\
min. Pol. & $60 \mu \mathrm{m}$ & $8.63^{3}$ & 6.93 \\
max. Pol. & $1300 \mu \mathrm{m}$ & $7.39^{3}$ & 6.63 \\
\hline
\end{tabular}

References: 1) Strom \& Strom (1994); 2) Luhman \& Rieke (1998); 3) Kenyon et al. (1990); 4) Luhman (2000); 5) Motte \& André (2001); 6) This paper, see Sect. 3.2.

appears to be systematically lower by a few percent compared to the data. Nonetheless, the trends are well reproduced as well as the observed maximum polarisation rates.

\section{Discussion}

\subsection{Reliability of the models}

We were able to fit a dust disc model to the observations of IRAS $04158+2805$ that matches the $I-, H$ - and $K$-band images as well as the SED from visual to far-infrared and the $I$-band polarisation rate at different object positions. Since our model uses simple assumptions, such as homogeneous spherical grains, power-law distributions (grain size, surface density, flaring) and no dust settling, these results provide a useful insight on the geometry of the circumstellar environment.

We fitted the observation well in terms of the width of the nebula, opening angle and peak-to-peak distance and brightness contrast (see Table 3 for all observables). The triangularity $t$ is well fitted for the $I$ - and $H$-band but for the $K$-band the model is too triangular. At the centre of the object, the model predicts a polarisation rate which is slightly below the observed value. While this probably indicates that our grain model needs refinement (either in its size distribution, composition or grain shape), the former may indicate that we need a more complex geometry either for the disc or for the emitting source. For instance, $K$-band emission from the inner parts of the disc might be an important source of photons, which is neglected in our calculations. To check this possibility we calculated a $K$-band model image that includes the disc emission as a source for scattered photons for our best-fitting model. It turns out that the star is so 
cool that, at the $0.5 \mathrm{AU}$ assumed inner radius, the dust reaches a temperature of only $400 \mathrm{~K}$. As a consequence, roughly $99 \%$ of all $K$-band photons emitted by the system come from the star and, therefore, the resulting images are unchanged if we include or neglect the disc emission. Vertical settling could also play a role, as well as the presence of a remnant halo that could be responsible for the roundish $4-5^{\prime \prime}$ structure best highlighted at $K$-band, where the extinction of the material located in front becomes much lower.

By comparing the $I$-band observed and modelled images, the shape of the counter nebula and the dark lane between the two bright areas do not coincide exactly. The model did not take into account the absorption of background light in the dust lane. The artificial noise of the model images was produced by adding Poisson noise to the scattered light images and by superposing position-independent Gaussian-noise. This does not reflect the real nature of the background since IRAS $04158+2805$ is illuminated by the large reflection nebulosity in the back. Therefore, we may expect a difference of the observation and the models at those wavelengths where the background light dominates the noise. In the $H$ - and $K$-bands this dominance is not visible in the observed images.

The model underpredicts the millimeter flux by a factor of 3 but uncertainties are large regarding dust opacities in this regime, typically by a factor of 5 . Also, the mm-data were obtained with an 11 arcsec beam and may suffer from background contamination, hence overestimate the true flux. Higher resolution millimeter data is needed to investigate this discrepancy further.

Nevertheless, one single model can describe accurately the scattered light images in three wavelength bands, the SED and the polarisation map of IRAS $04158+2805$. The method applied here is therefore generally valid and promising to find parameters for relatively simply structured dust discs.

\subsection{Gas to dust ratio}

From our model, we can easily derive the column density $\sigma_{\text {dust }}$ by integrating the dust density structure along our line of sight to the central source. We find $\sigma_{\text {dust }}=3.3_{-1.2}^{+1.8} \times 10^{-4} \mathrm{~g} \mathrm{~cm}^{-2}$. From $N_{\mathrm{H}}$ we obtain a gas column density $\sigma_{\text {gas }}=7.2_{-3.0}^{+4.8} \times 10^{-2} \mathrm{~g} \mathrm{~cm}^{-2}$ by assuming interstellar abundances (Morrison \& McCammon 1983). This provides an estimate of the total gas-to-dust ratio in a protoplanetary disc, along the line of sight that grazes the disc top layers in this case. To our knowledge this is the first time it is obtained directly using $N_{\mathrm{H}}$ rather than $N_{\mathrm{CO}}$ :

$$
\frac{\sigma_{\mathrm{gas}}}{\sigma_{\mathrm{dust}}}=220_{-150}^{+170} .
$$

While uncertainties on this ratio are still substantial, this illustrates a robust method to derive dust-to-gas ratios in protoplanetary discs. Although the value we find is compatible with the standard value of 100 usually assumed in this context, our result might suggest a slightly higher value in the top layers of the disc. This is a potentially important result that calls for further investigations in more sources.

In any case, we do find an upper limit to the gas-to-dust ratio (albeit not a lower limit) of $\approx 560(2 \sigma)$, which provides clear evidence that the disc cannot be strongly (e.g. by an order of magnitude) depleted of dust along the line of sight to the star. Such dust depletion would be expected if strong settling of dust toward the disc midplane had occurred.

\subsection{IRAS 04158+2805: a classical T Tauri star with a large disc?}

We have shown that we can well reproduce all observational properties of IRAS04158 with a simple model of a nearly edgeon disc, i.e., that of a Class II source without substantial circumstellar envelope. This result confirms prior classifications by e.g. Park \& Kenyon (2002) or Kenyon \& Hartmann (1995). The flat SED of IRAS $04158+2805$ can be interpreted in terms of a high inclination to the line of sight instead of invoking a highly embedded source, as a protostar would be. This work demonstrates the importance of understanding the circumstellar geometry to assess the nature of the central source. Here, an edge-on disc blocks our direct view towards the central object, flattening the shape of the SED. It is known that a classical T Tauri star can have a rising near- to mid-infrared SED. But only a few mimic an embedded source, as is the case for IRAS 04158+2805. Most sources such as HH30 (see, e.g., Wood et al. 2006), HK Tau B, or HV Tau C, do have a declining near- to mid-IR SED, and the second peak from thermal emission is found only at longer wavelengths.

Interestingly, IRAS $04158+2804$ is just above the substellar limit, based on its spectral type. If our model is correct, it hosts a large massive disc implying that some of the lowest-mass T Tauri stars, at least in Taurus, can be surrounded by $\sim 1000$ AU-radius discs. This object likely formed from the collapse of a small prestellar core and is very unlikely to have undergone any violent dynamical interaction, such as an ejection from an unstable multiple system (e.g. Reipurth \& Clarke 2001). This result adds support to the idea that the ejection scenario is not the only mode to form VLM T Tauri stars and brown dwarfs.

Also, considering the probable mass of the central star $\sim 0.1-0.2 M_{\odot}$, the total dust-disc mass we derive $\left(1-2 \times 10^{-4} M_{\odot}\right)$ and the gas-to-dust ratio we derived $(\sim 220$, assuming it is true throughout the disc and not only along our line of sight), this implies a total disc/star mass ratio of $\sim 0.1-0.2$. In other words, the disc is close to the limit against gravitational instability. Because collapse in these gravitationally unstable discs is one suggested mode for planet formation, it is of great importance to study IRAS $04158+2805$ further: the estimation of both the gas and the dust masses need to be refined. The gas mass estimation can be improved by much deeper X-ray observations while the dust mass can be obtained better with models witch use more free parameters and which are directly fitted on the images, not just a handful of observables.

\section{Conclusion}

In this paper we presented a multiwavelength study of IRAS $04158+2805$ and its circumstellar environment. We modelled the shape and brightness profiles of the reflection nebulosity in three optical and NIR bands ( $I$-, $H$ - and $K$-band) with MCFOST, a Monte Carlo polarised radiative transfer code, and found a good agreement between the model and the data. Many parameters of the final model are well constrained (e.g., inclination, $\left.a_{\max }, M_{\text {dust disc }}\right)$ while a few remain poorly determined. The scale height $h$ and $\beta$ are degenerate but the pair $h-\beta$ is relatively constrained.

The disc model parameters used to match the data are listed in Table 2. They are $2 \mu \mathrm{m}$ for the maximum dust grain size, $1.5 \times 10^{-4} M_{\odot}$ for the dust disc mass, $1120 \mathrm{AU}$ for the outer disc radius, 1.2 for the exponent $\beta$ of the flaring law, -1.0 for the 
exponent $\alpha$ of the radial dust density law, a scale height of $8 \mathrm{AU}$ at a radius of $50 \mathrm{AU}$ and an inclination of $62.7^{\circ}$.

The best model fits the observed SED well. However, it falls slightly short of producing the right amount of $1.3 \mathrm{~mm}$ continuum flux by about a factor of 3 . The model also reproduces reasonably the observed $I$-band polarisation behaviour along the symmetry axis and ridges of the Northern nebula.

Combining dust disc models with X-ray spectroscopy allowed probing the gas-to-dust ratio in the disc. We found a value of $220_{-150}^{+170}$ which is compatible with the ISM value and the value generally assumed in protoplanetary discs.

According to its spectral type, IRAS $04158+2805$ has a mass slightly above the substellar limit. Clearly, stars with such a low mass keep being formed by accretion from a circumstellar disc whose properties do not seem to differ significantly from those of their more massive young counterparts. It would be interesting to push the search further for disc images around less massive objects, located well into the substellar regime.

Acknowledgements. This work is based in part on archival data obtained with the SPITZER Space Telescope, which is operated by the Jet Propulsion Laboratory, California Institute of Technology under a contract with NASA. Support for this work was provided in part by an award issued by JPL/Caltech and in part by Programme National de Physique Stellaire (PNPS) of CNRS/INSU (France). We are grateful to Sylvain Bontemps for obtaining the CFHTIR images presented here and to Jérome Bouvier for performing their basic data reduction. The CXC X-ray Observatory Center is operated by the Smithsonian Astrophysical Observatory for and on behalf of the NASA under contract NAS8-03060.

\section{References}

Anders, E., \& Grevesse, N. 1989, Geochim. Cosmochim. Acta, 53, 197 Arnaud, K. A. 1996, in Astronomical Data Analysis Software and Systems V, ed. G. H. Jacoby, \& J. Barnes (San Francisco: ASP), ASP Conf. Ser., 101, 1 Baraffe, I., Chabrier, G., Allard, F., \& Hauschildt, P. H. 1998, A\&A, 337, 403 Güdel, M. 2004, A\&A Rev., 12, 71

Houck, J. R., Roellig, T. L., van Cleve, J., et al. 2004, ApJS, 154, 18 Kenyon, S. J., \& Hartmann, L. W. 1995, ApJS, 101, 117

Kenyon, S. J., Hartmann, L. W., Strom, K. M., \& Strom, S. E. 1990, AJ, 99, 869 Leroy, J.-L. 1993, A\&A, 274, 203

Leroy, J.-L. 1999, A\&A, 346, 955

Luhman, K. L. 2000, ApJ, 544, 1044

Luhman, K. L. 2006, ApJ, 645, 676

Luhman, K. L., \& Rieke, G. H. 1998, ApJ, 497, 354

Mathis, J. S., \& Whiffen, G. 1989, ApJ, 341, 808

Monin, J.-L., Ménard, F., \& Peretto, N. 2006, A\&A, 446, 201

Morrison, R., \& McCammon, D. 1983, ApJ, 270, 119

Motte, F., \& André, P. 2001, A\&A, 365, 440

Patat, F., \& Romaniello, M. 2006, A\&A, 118, 146

Pinte, C., Ménard, F., Duchêne, G., \& Bastien, P. 2006, A\&A, 459, 797

Reipurth, B., \& Clarke, C. 2001, AJ, 122, 432

Schegerer, A., Wolf, S., Voshchinnikov, N. V., et al. 2006, A\&A, 456, 535

Schneider, G., Silverstone, M. D., Hines, M. D., et al. 2005, in Protostars \& Planets V, HST High-Contrast Imaging of Circumstellar Disks with Optical/Near-IR Coronagraphy

Stapelfeldt, K. R., Padgett, D. L., Ménard, F., et al. 2003, in Star Formation at High Angular Resolution, IAU Symp., 221, 276

Starr, B. M., Doyon, R., Beuzit, J.-L., et al. 2000, SPIE, 4008, 999

Strom, K. M., \& Strom, S. E. 1994, ApJ, 424, 237S

Telleschi, A., Güdel, M., Briggs, K. R., et al. 2007, A\&A, 468, 425

Watson, D. M., Kemper, F., Calvet, et al. 2004, ApJS, 154, 391

Wood, K., Lada, C. J., Bjorkman, J. E., et al. 2002, ApJ, 567, 1183 